\title{
Factors that influence the survival of unstable pelvic fracture in the acute phase
}

\author{
Komang A. Irianto, Muhammad Z. Chilmi, Gana Adyaksa
}

Departement of Orthopedic and Traumatology, Faculty of Medicine, Universitas Airlangga, Dr. Soetomo General Hospital, Surabaya, Indonesia

\section{ABSTRACT}

Background: The fatality rate of unstable pelvic fracture is still high (20-50\%) due to acute phase hemorrhage or late multiple organ failure. The assurance for $100 \%$ survival in acute phase had been reported if the emergency room team have the right technique, right timing, and right protocol. The aim of this study was to evaluate and to date factors that influence the nonsurvival unstable pelvic fracture within $2 \times 24$ hours.

Methods: This retrospective study was conducted in emergency unit of Dr. Soetomo General Hospital Surabaya during 20122016 for unstable pelvic fracture with unstable hemodynamic, without concomitant head or thoracic injury and admitted within 4 hours after the incident. All patients were handled according to the protocol for resuscitation and C-clamp insertion immediately. $\mathrm{X}$-ray was taken after the insertion to check the C-clamp position and to confirm the diagnosis. The $2 \times 24$-hour data were taken whether the patient survive or not survive, including mean arterial pressure (MAP) pre-and post, heart rate (HR) pre-and post, and initial hemoglobin and platelet count. Data were analyzed with independent t-test and multiple regression analysis.

Results: Twenty two patients were recorded as the inclusive criteria, separated into 17 survivors and 5 non-survivors. They were comprised of 14 males and 8 female patients, with an average of 31.9 \pm 15.3 years old. The MAP post, HR pre-and post, and platelet count were significantly different. The factor that influenced the survival within $2 \times 24$ hours was the MAP post C-clamp $(>70 \mathrm{mmHg})(\mathrm{p}<0.05)$.

Conclusion: The acute phase $2 \times 24$-hour outcome after C-clamp insertion and resuscitation was significantly influenced by MAP post C-clamp and resuscitation.

Keywords: C-clamp, MAP post-resuscitation, unstable pelvic fracture

pISSN: 0853-1773 • eISSN: 2252-8083• https://doi.org/10.13181/mji.v27i1.2078• Med J Indones. 2018;27:33-7

- Received 26 Jun 2017 • Accepted 28 Dec 2017

Corresponding author: Komang A. Irianto

komang168@yahoo.com

Copyright @ 2018 Authors. This is an open access article distributed under the terms of the Creative Commons Attribution-NonCommercial 4.0 International License (http://creativecommons.org/licenses/by-nc/4.0/), which permits unrestricted non-commercial use, distribution, and reproduction in any medium, provided the original author and source are properly cited. 
Unstable pelvic fracture is a life-threatening injury related to hemodynamic instability due to acute blood loss from retroperitoneal bleeding in the first 24 hours, and later to multisystem organ failure. ${ }^{1-3} \mathrm{C}$-clamp is the favorable and favorite tool in stabilizing the pelvic ring injury and controlling the bleeding in retroperitoneal area. The survival rate of C-clamp insertion was reported increased from $57 \%$ during $1996-2001$, to $71 \%$ in $2002-$ 2008 , then $81 \%$ by 2015 ; after developing the protocol by including retroperitoneal pelvic packing and angio-embolization. ${ }^{3}$ There is always the issue of whether the hemorrhage could be controlled effectively without losing the time by doing several protocols. The best procedure choice and sequence in the initial management was to treat immediately with C-clamp in the emergency department $(100 \%$ survival rate). Immediate C-clamp insertion alone is the best methods comparing to others such as C-clamp followed by angiography ( $91 \%$ survival rate), C-clamp followed by laparotomy plus minus angiography ( $82 \%$ survival), and immediate laparotomy or angiography before C-clamp ( $0 \%$ survival rate). ${ }^{4}$

Postmortem angiography and dissections after pelvic trauma showed that posterior venous bleeding and cancelous bone in the fracture were the massive leakage of the bleeding point, while arterial bleeding was present in less than $10 \%$. Several techniques to stop the bleeding, stabilized the hemodynamic and the mechanic stabilization were established and applied, yet they were not competitive but complementary. Either one technique or protocol is effective if executed with the right technique, right timing, in the right patient. ${ }^{5}$ Since pelvic fracture is only $3 \%$, limited patients and limited cases make it hard for the orthopedic trainee to be experienced with the device. The training for orthopedic surgeon is an obligation. Monitoring and evaluation of the outcome would give feed back to the institution. ${ }^{4-7}$

There was still no evaluation for the efficacy of this procedure in treating unstable pelvis. The aim of this study was to evaluate and to date factors that influenced the non-survival unstable pelvic fracture within $2 \times 24$-hours.

\section{METHODS}

This retrospective study of all unstable pelvic fracture with unstable hemodynamic in emergency unit of the Orthopedic Department, Dr. Soetomo General Hospital Surabaya, Indonesia during 2012-2016 has been approved by the Institutional Review Board. Data were collected and grouped into survivor (S group) and non-survivor (NS group) within the $2 \times 24$ hour acute phase. The inclusive criteria are the injury within 4 hours before admission, and without any associated head or thoracic injury. Data were sorted including initial hemodynamic statues based on the primary survey according to advance trauma life support (ATLS) protocol in triage.

Unstable pelvic fracture was diagnosed clinically by assessing antero-posterior compression, lateral compression, and vertical shear signs found. ${ }^{2}$ Together with unstable hemodynamic (low mean arterial pressure (MAP), high heart rate (HR), low hemoglobin, and low platelet count); those conditions were managed by hemodynamic resuscitation and
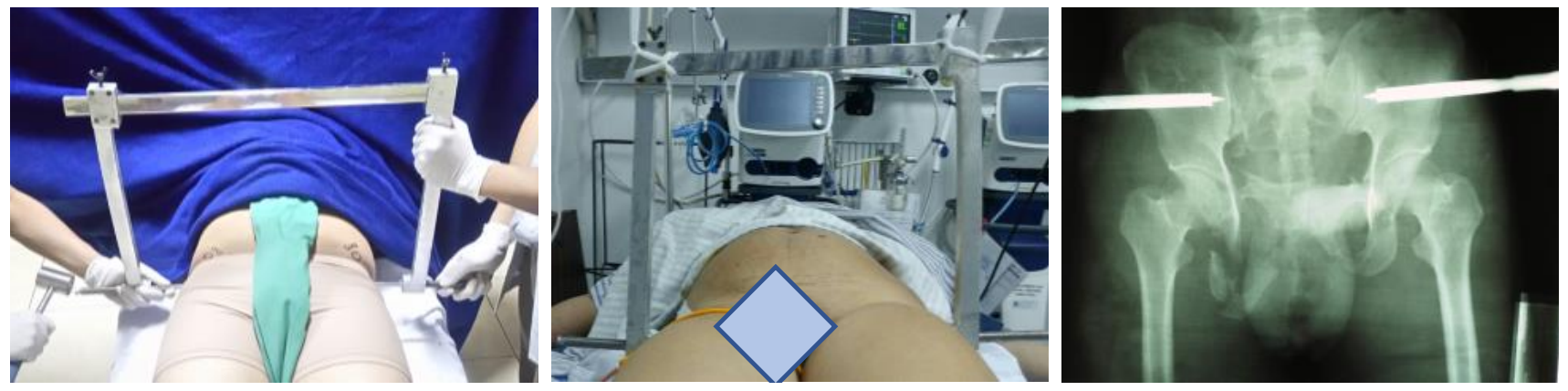

Figure 1. C-clamp insertion under local anesthesia in ER. Two pins were each screwed at sacroiliac left and right (inserting point is the intersection between vertical line passing Spina Iliac Anterior Superior and horizontal line through axis of the femur). Both pin compressed simultaneously to restore the pelvic ring and reduced sacroiliac disruption 
immediate C-clamp insertion in the emergency unit by orthopedic surgeon. The C-clamp assembly took 10 minutes then confirmed by X-ray for the accuracy of pin placement and frame assembly. ${ }^{8}$ C-clamp was a temporary fixation, whenever needed, internal fixation should replace it. The quick, easy, and safe technique giving access for further laparotomy or further adjunct evaluation i.e. magnetic resonance imaging (MRI) (Figure 1).

After resuscitation, the hemodynamic statuses were examined based on patients' latest conditions when leaving the resuscitation room (prior to emergency surgery, or prior to being transferred to definitive care). Hemoglobin and platelet count post resuscitation and C-clamp insertion were not taken because of the interference of transfusion. Descriptive and comparison analytic using independent t-test was used to find out the different profile between the survivor and the non-survivor patients. The significant factors were then put into multiple regression analysis to note the possible most influential factor that held the chance for survival.

\section{RESULTS}

There was a total of 22 patients with unstable pelvic fracture and unstable hemodynamic that met the inclusive criteria. They were comprised of 14 males and 8 female patients, with an average age of $31.9 \pm 15.3$ years old. The major modes of injury were traffic accident $(n=18)$ and falling from a height $(n=4)$. A total of 5 patients died within 2x24 hours (Non-Survivor/ NS group) and 17 patients survived (Survivor/ S group), comprised of $77.3 \%$ survival rate.
Data of Hemodynamic status within $2 \times 24$-hour evaluation of the $S$ group and NS group (Table 1) shows significant different in MAP post resuscitation to increase to above the normal value $(>70 \mathrm{mmHg}$ ). The outcome value in the $\mathrm{S}$ group elevated to $81.9 \pm 7.2 \mathrm{mmHg}$, while the NS group was below the normal value $(63.0 \pm 7.8 \mathrm{mmHg})$. Heart rate before and after C-Clamp with resuscitation were improved both in the $S$ group and NS group, yet the initial HR in the NS group were worse $(127.6 \pm 3.2)$ and still very high afterward (120.8 \pm 6.4$)$; while the $S$ group showed better outcome (110.7 \pm 9.7$)$. The mean hemoglobin count before C-Clamp with resuscitation were not differ within both the $S$ group $(7.6 \pm 2.1 \mathrm{mg} \%)$ and NS group $(5.9 \pm 1.8$ $\mathrm{mg} \%$ ), while the thrombocyte count was significantly differed $(206.0 \pm 96.0$ compared to $71.6 \pm 80.2$ ). Evaluation with multiple regression analysis for four significant different values within the $S$ and NS group revealed that only MAP post C-clamp with resuscitation is the possible most influential factor that hold the chance for survival (Table 1).

\section{DISCUSSION}

The protocol for unstable pelvis in our hospital is C-clamp insertion directly while stabilizing the hemodynamic status. The reason is because it is simple, easy, and technically fast (10 minutes of assembly) ${ }^{8}$; and under the circumstances that angiography is inaccessible in the emergency unit. In this study, the time interval for monitoring was limited for acute phase, where the unstable hemodynamic was likely caused by hemorrhage and exsanguinous. ${ }^{9,10}$ It is the general acceptance in ATLS that in the acute phase, the

Table 1. Hemodynamic Status evaluated in 2x24 hours

\begin{tabular}{|c|c|c|c|c|c|}
\hline & All Patients & $S(n=17)$ & NS $(n=5)$ & p-values* & p-values ${ }^{\dagger}$ \\
\hline MAP initial (mmHg) & $61.5 \pm 7.1$ & $62.7 \pm 6.5$ & $57.2 \pm 8.2$ & 0.12 & 0.808 \\
\hline MAP post C-clamp (mmHg) & $77.2 \pm 10.8$ & $81.9 \pm 7.2$ & $63.0 \pm 7.8$ & $0.00^{*}$ & $0.009^{*}$ \\
\hline HR initial & $122.6 \pm 10.0$ & $120.6 \pm 6.9$ & $127.6 \pm 3.2$ & $0.04^{*}$ & 0.309 \\
\hline HR post C-clamp & $113.0 \pm 9.9$ & $110.7 \pm 9.7$ & $120.8 \pm 6.4$ & $0.04^{*}$ & 0.320 \\
\hline Hb initial (g\%) & $7.2 \pm 2.0$ & $7.6 \pm 2.1$ & $5.9 \pm 1.8$ & 0.13 & 0.324 \\
\hline Platelet initial & $172.4 \pm 105.2$ & $206.0 \pm 96.0$ & $71.6 \pm 80.2$ & $0.01^{*}$ & 0.149 \\
\hline
\end{tabular}

$\mathrm{MAP}=$ mean arterial pressure; $\mathrm{HR}=$ heart rate; $\mathrm{Hb}=$ hemoglobin; $\mathrm{S}=$ survivor; $\mathrm{NS}=$ non-survivor; $\mathrm{p}$-value*= data was tested by independent $t$-test; $p$-value ${ }^{\dagger}=$ data was tested by multi-regression analysis. Numerical data was presented in Mean $\pm S D$ 
hemodynamic stability plays a major role in the total outcome of hemorrhage cases. ${ }^{10}$ Damage control orthopedic is needed in unstable pelvic fracture. ${ }^{11-13}$ The Malgaigne Era $\left(19^{\text {th }} \text { century }\right)^{12}$ start of a modern history of pelvic fracture, closed reduction to stabilize while stopping the bone hemorrhage as well, has proven to elevate the survival rate. Early fixation of skeletally unstable pelvic fractures could reduce the hospital stay, long-term disability, decreased blood loss, fewer complication, and better survival. ${ }^{9}$

Is the $22.7 \%$ mortality in our report may be caused by the incompetence of technically C-clamp insertion, then how do we know that the C-clamp does not effectively stop the exsanguinous? The X-ray after the insertion only roughly confirmed the accuracy of the pin, yet it could not foresee the probably inadequate tamponade in stopping the exsanguinous. ${ }^{10,14}$ The clinical parameter (heart rate, mean arterial pressure) and physiological parameter (lactate levels, hemoglobin, hematocrit) could show the ongoing hemodynamic status. ${ }^{14-16}$

Tiemann et $\mathrm{al}^{16}$ showed that Hemoglobin and Hematocrit were not sensitive and could lead to fatal perception of the true hemodynamic status since those two parameters did not have significant correlation with cellular perfusion and regional tissue oxygenation. Blood pressure (systolic) has long been sided away since MAP (mean arterial pressure) showed true hemodynamic status. ${ }^{14-16}$ This study showed that initial MAP did not differ in all patients since they all admitted in poor hemodynamic condition. Albeit the resuscitation and C-clamp insertion, the uncontrolled MAP afterward differed the outcome of the patient. The heart rate before and after the resuscitation could be an alarm, but it was not sensitive enough and influenced by other non-hemodynamic condition such as psychologic, accompanying pain, or hormonal. A scoring system was built to predict the prognostic factor of massive hemorrhage of an unstable pelvic trauma; where the only hemodynamic and physiologic factor included is Lactate blood level. Other center also applied the lactate level in the protocol to give picture of the end result of the unstable hemodynamic. ${ }^{1-4,17-19}$

The late mortality of unstable pelvic injury caused by multiple organ failure owing to intractable hemodynamic shock. ${ }^{14,18-20}$ The main bleeding source was not the rupture artery, but the exsanguinous and the consuming of the coagulopathy components were. ${ }^{14,20}$ The low thrombocyte count in this study did not have any association with the hematologic problem. The significant difference of the thrombocyte count between the survivors and the non-survivors might be attached to the consuming coagulopathy in massive hemorrhage. Unfortunately, this study did not have other coagulant components.

In conclusions, the probability of survive in acute phase ( $2 \times 24$ hours $)$ after C-clamp insertion and resuscitation is affected by the postoperative mean arterial pressure.

\section{Conflict of interest}

The authors affirm no conflict of interest in this study.

\section{Acknowledgement}

The author would like to thank all staff of Department of Orthopedic and Traumatology Dr. Soetomo General Hospital for preparing the database of the patients.

\section{REFERENCES}

1. Wang H, Robinson RD, Moore B, Kirk AJ, Phillips JL, Umejiego J, et al. Predictors of early versus late mortality in pelvic trauma patients. Scand J Trauma Resusc Emerg Med. 2016;24:27.

2. Rudloff MI, Triantafillou KM. Management of pelvic ring injuries in unstable patients. Orthop Clin N Am. 2016;47(3):551-63.

3. Lau J, Chui KH, Ip KC, Li W. Improved Survival with a standardized multidisciplinary 3 in-1 pelvic damage control protocol for hemodynamically unstable pelvic fracture. J Emerg Trauma Care. 2016;1:1.

4. Abrassart S, Stern R, Peter R. Unstable pelvic ring injury with hemodynamic instability: What seems the best procedure choice and sequence in the initial management? Orthop Traumatol Surg Res. 2013;99:175-82.

5. Rommens PM, Hofmann A, Hessmann MH. Management of acute hemorrhage in pelvic trauma: an overview. Eur J Trauma Emerg Surg. 2010;36(2):91-9.

6. Wiznia DH, Swami N, Kim CY, Leslie MP. A method of using a pelvic C-clamp for intraoperative reduction of a zone 3 sacral fracture. Case reports in Orthopaedics. Hindawi Publishing Corporation. 2016;2016:8586517.

7. Petrisor BA, Bhandari M. Injuries to the pelvic ring: incidence, classification, associated injuries and mortality rates. Curr Orthopaed. 2005;19(5):327-33. 
8. Hildegard H, Balogh ZJ. Single training session for first time pelvic C-clamp users: correct pin placement and frame assembly. Injury. 2012;43(4):436-9.

9. Latenser BA, Gentilello LM, Tarver AA, Thalqott JS, Batdorf JW. Improved outcome with early fixation of skeletally unstable pelvic fractures. J Trauma. 1991;31(1):25-31.

10. Keel M, Otmar T. Acute management of pelvic ring fractures. Curr Orthopaed. 2005; 19(5):334-44.

11. Giannooudis PV, Pape HC. Damage control orthopaedics in unstable pelvic ring injuries. Injury. 2004;35(7):671-7.

12. Philip FS, Hammerberg EM. History of pelvic fracture management: a review. World J Emerg Surg. 2016;11:18.

13. Giannoudis PV, Giannoudi M, Stavlas P. Damage control orthopaedics: lessons learned. Injury. 2009;40(Suppl 4);S47-52.

14. Lustenberger $\mathrm{T}$, Meier $\mathrm{C}$, Benninger, Lenzlinger PM, Keel MJ. C-clamp and pelvic packing for control of hemorrhage in patients with pelvic ring disruption. J Emerg Trauma Shock. 2011;4(4):477-82.
15. Hsu JM, Yadev S, Faraj S. Controlling hemorrhage in exsanguinating pelvic fractures: utility of extraperitoneal pelvic packing as a damage control procedure. Int J Crit Illn Inj Sci. 2016;6(3):148-52.

16. Tiemann AH, Boehme J, Jonsten C. Emergency treatment of multiple injured patients with unstable disruption of the posterior pelvic ring by using the C-clamp. Eur J Trauma. 2005;31:244-51.

17. Ohmori T, Matsumoto T, Kitamura T, Tamura R, Tada $\mathrm{K}$, Inoue $\mathrm{T}$, et al. Scoring system to predict hemorrhage in pelvic ring fracture. Orthop Traumatol Surg Res. 2016;102(8):1023-28.

18. Filiberto DM, Fox AD. Preperitoneal pelvic packing: techniques and outcome. Int J Surg. 2016;33(Pt B):222-4.

19. Papakostidis C, Giannoudis PV. Pelvic injuries with hemodynamic instability: efficacy of pelvic packing, a systematic review. Injury. 2009;40(Suppl 4):S53-61.

20. Boral BM, Williams DJ, Boral LI. Disseminated intravascular coagulation. Am.J.Clin.Pathol. 2016;146(6):670-80. 